Molecules 2004, 9, 913-921

molecules

ISSN 1420-3049

http://www.mdpi.org

\title{
Synthesis and Biological Activities of Novel Triazole Compounds Containing 1,3-Dioxolane Rings
}

\section{Liang-Zhong Xu, Shu-Sheng Zhang*, Shu-Yan Niu, Yong-Qi Qin, Xue-Mei Li and Kui Jiao}

College of Chemistry and Molecular Engineering, Qingdao University of Science and Technology, Qingdao, 266042, P. R. China

* Author to whom correspondence should be addressed; E-mail: zhangshush@public.qd.sd.cn; Tel: (+86)-532-4022750; Fax: (+86)-532-4023927

Received: 29 March 2004 / Accepted: 4 August 2004 / Published: 30 November 2004

\begin{abstract}
Thirteen new triazoles containing 1,3-dioxolane rings were synthesized and their identities confirmed by means of IR, NMR, MS, elemental analysis and X-ray crystallography. The results of preliminary biological tests show that all of these compounds possess some fungicidal and plant growth regulant activities.
\end{abstract}

Keywords: 1,2,4-Triazole; 1,3-Dioxolane; Biological activities; Fungicide; Plant growth regulant.

\section{Introduction}

Many triazole compounds have good fungicidal and plant growth regulating activities [1-5]. In particular, triazole compounds containing 1,3-dioxolanes have been shown to have remarkable preventative and control activities for a variety of plant diseases [6]. Propiconazole and difenoconazole are two important representatives of this class, especially the latter, which has been used as the most efficient triazole fungicide in the control of some common plant diseases. The key intermediates for its synthesis, however, are not easily obtained, which makes the costs of production and application too high. Following the concept of bioisosterism [7-8], we have now synthesized ten novel 1,2,4-triazole compounds containing 1,3-dioxolane rings, 3a $\sim 3 \mathbf{j}$, using difenoconazole as the lead compound. In addition, according to empirical data [9], the presence of an exposed triazole ring is conducive to 
improvements of the biological activity. To explore this idea three new compounds $5 \mathbf{a} \sim 5 \mathbf{c}$ containing 1,2,4-triazole-substituted 1,3-dioxolanes were also synthesized. The synthetic routes used are shown in Scheme 1.<smiles>CCC1COC(Cn2cncn2)(c2ccc(Cl)cc2Cl)O1</smiles>

propiconazole

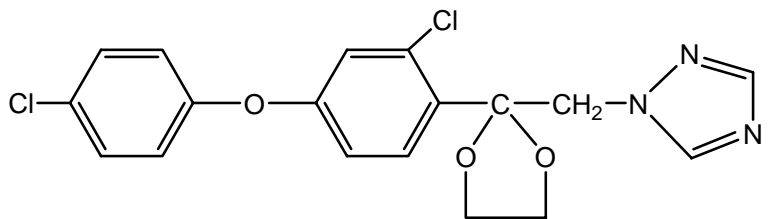

difenoconazole

\section{Scheme 1}

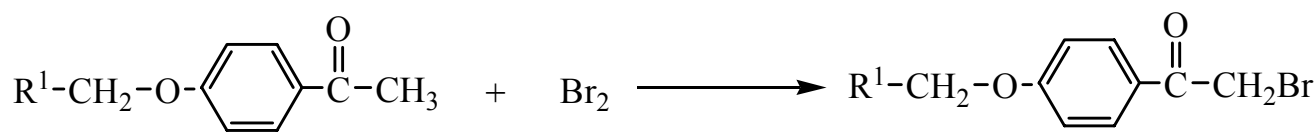

1

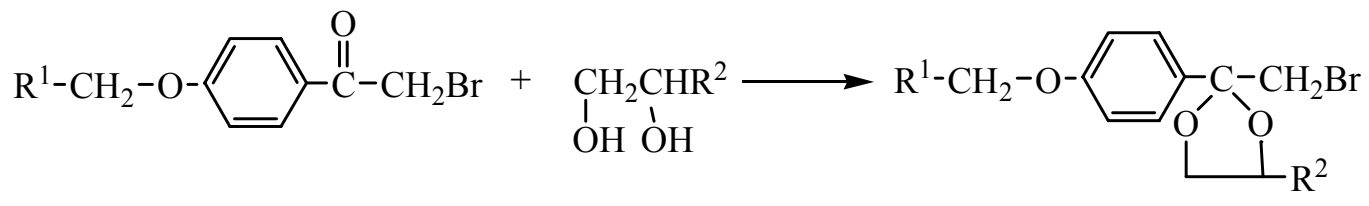

2

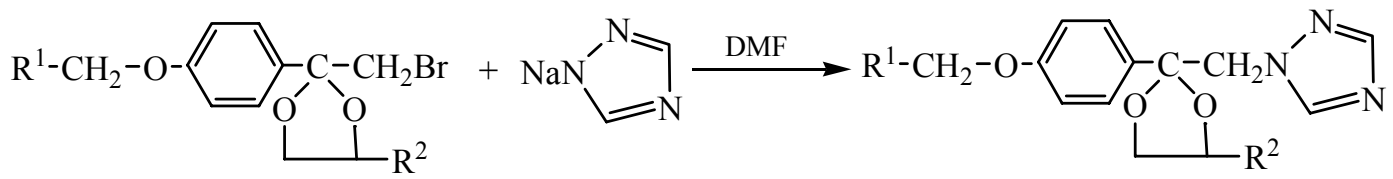

3

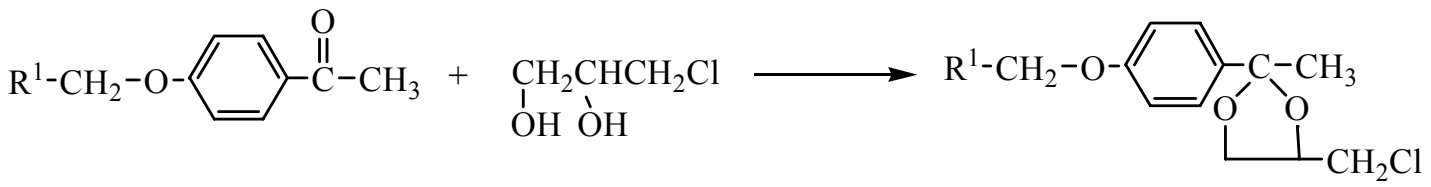

4<smiles>[R]COc1ccc(C2(C)OCC(CCl)O2)cc1</smiles> 


\section{Results and Discussion}

\section{Characterization of the target compounds}

Compounds $\mathbf{3 a} \sim \mathbf{3} \mathbf{j}$ and $\mathbf{5 a} \sim \mathbf{5} \mathbf{c}$ were identified by their CHN analysis, IR, ${ }^{1} \mathrm{H}-\mathrm{NMR}$, EA and MS fragmentation pattern data. The measured values in the elemental analyses were consistent with the corresponding calculated ones. The IR spectra display medium or weak absorption bands for the benzene and triazole rings at around $3100 \mathrm{~cm}^{-1}\left(v_{\mathrm{C}-\mathrm{H}}\right)$, while the two $\mathrm{C}-\mathrm{O}-\mathrm{C}$ ether bonds give relatively strong absorption bands at around $1170 \mathrm{~cm}^{-1}$ and $1140 \mathrm{~cm}^{-1}$. The absorption for the $\mathrm{PhCH}_{2}-\mathrm{O}-\mathrm{Ph}$ ether bond appears at around $1240 \mathrm{~cm}^{-1}$. In the ${ }^{1} \mathrm{H}-\mathrm{NMR}$ spectra of compounds $\mathbf{5 a} \sim \mathbf{5} \mathbf{c}$ the signals of the two protons of the $\mathrm{CH}_{2}$ group connecting the triazoles with the 1,3-dioxolane appear as a multiplet at around $3.8 \mathrm{ppm}$.. It is believed that this is due to the fact they are attached to an asymmetrical carbon atom, which makes the magnetic environments of the two $\mathrm{CH}_{2}$ group protons different. When $\mathrm{R}^{2}$ is $\mathrm{CH}_{3}$, the protons of the $\mathrm{CH}_{3}$ group and triazole ring are all split into two sets of peaks. The molecular ion peaks of the title compounds examined by mass spectrometry are very weak, but all of the key fragment ion peaks appear. Additional proof for the proposed structures was provided by single crystal X-ray diffraction of a representative compound (Figure 1) [10].

Figure 1. Molecular structure of compound $\mathbf{3 g}$ with the atomic numbering scheme.

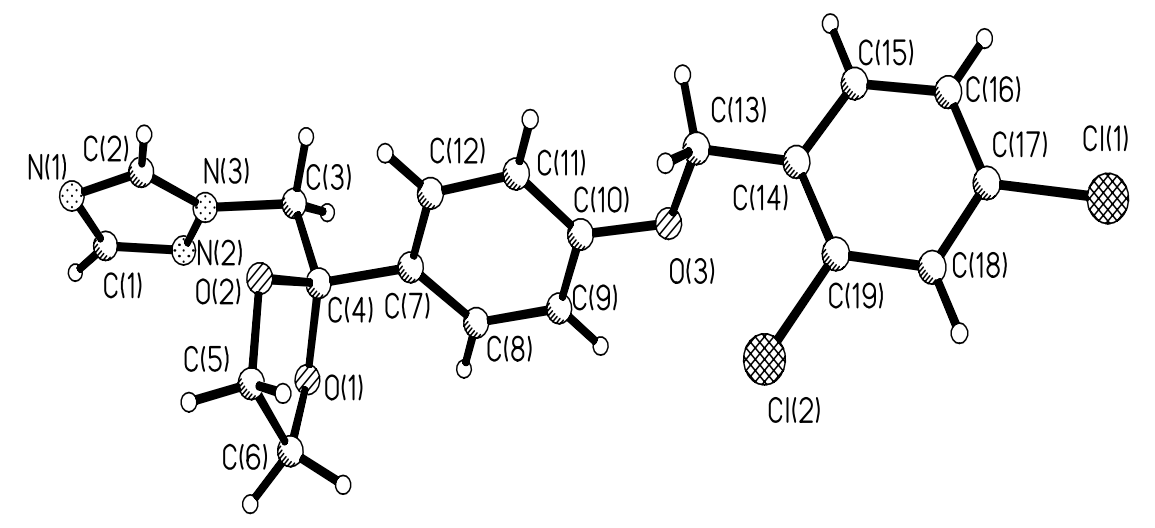

\section{Biological evaluation}

The antifungal activities of the synthesized compounds against Gibberella zeae, Alternaria solani, Phoma asparagi, Physalospora pircola and Cercospora arachidicola were determined by the "contained poison in the medium" method [11]. Plant-growth regulatory activities of the target compounds on wheat coleoptile elongation, cucumber cotyledon rooting, rape hypocotyls inhibition and growth of cucumber cotyledon were tested by the methods mentioned in the literature [12 15]. 
The data in Table 1 show that most of the target compounds have some fungicidal activity. Overall they exhibit better efficiency against $P$. Pircolae, particularly $\mathbf{3 a}$, $\mathbf{3} \mathbf{i}$ and $\mathbf{3} \mathbf{j}$, with inhibition rates at $50 \mathrm{mg} / \mathrm{L}$ reaching $77.8 \%, 84.1 \%$ and $84.1 \%$, respectively. As far as the relationships between structure and the activity are concerned, when the $\mathrm{R}^{1}$ group is 2-chlorophenyl, the compounds have more comprehensive fungus-inhibiting properties. All thirteen target compounds have plant-growth regulatory activity. They show inhibiting activity towards wheat coleoptile elongation, with rates ranging from 3.1\% 22.5\%. Most of them have promoting effects towards rooting of cucumber cotyledon, with promoting rates reaching 3.1\% 69.3\%. All the compounds displayed much less promoting activity towards growth of cucumber cotyledon. The highest promoting rate was $9.4 \%$.

Table 1. The fungicidal and plant growth regulatory activities of compounds $\mathbf{3}$ and $\mathbf{5}$

\begin{tabular}{|c|c|c|c|c|c|c|c|c|c|}
\hline \multirow[b]{2}{*}{$\begin{array}{l}\text { Compound } \\
\text { No. }\end{array}$} & \multicolumn{5}{|c|}{ Fungicidal activities (50mg/L, inhibition \%) } & \multicolumn{4}{|c|}{ Plant-growth regulatory activities (\%) at $10 \mathrm{mg} / \mathrm{L}$} \\
\hline & $\begin{array}{l}\text { Gibberella } \\
\text { zeae }\end{array}$ & $\begin{array}{l}\text { Alternaria } \\
\text { solani }\end{array}$ & $\begin{array}{l}\text { Phomaas } \\
\text { paragi }\end{array}$ & $\begin{array}{l}\text { Physalospora } \\
\text { pircola }\end{array}$ & $\begin{array}{l}\text { Cercosporaa } \\
\text { rachidicola }\end{array}$ & $\begin{array}{l}\text { Wheat } \\
\text { coleoptile } \\
\text { elongation }\end{array}$ & $\begin{array}{l}\text { Cucumber } \\
\text { Cotyledon } \\
\text { rooting }\end{array}$ & $\begin{array}{l}\text { Rape } \\
\text { hypocotyls } \\
\text { inhibition }\end{array}$ & $\begin{array}{l}\text { Cucumber } \\
\text { cotyledon } \\
\text { growth }\end{array}$ \\
\hline $3 a$ & 50.0 & 71.0 & 90.9 & 77.8 & 43.8 & -22.5 & 61.2 & -29.7 & -6.3 \\
\hline $3 \mathbf{b}$ & 40.0 & 67.7 & 88.9 & 71.4 & 43.8 & -20.2 & 53.2 & -24.9 & 0 \\
\hline $3 c$ & 5.0 & 25.8 & 11.8 & 28.9 & 28.6 & -5.4 & 2.1 & -19.7 & 3.1 \\
\hline 3d & 25.0 & 38.7 & 41.2 & 57.8 & 28.6 & -3.1 & -8.6 & -16.3 & -1.6 \\
\hline $3 \mathbf{e}$ & 20.0 & 41.9 & 17.6 & 40.0 & 14.3 & -13.2 & 69.3 & -24.5 & 1.6 \\
\hline $3 f$ & 26.9 & 0 & 0 & 44.6 & 22.2 & -7.4 & -39.7 & -24.9 & 3.1 \\
\hline $3 g$ & 7.7 & 19.0 & 7.7 & 24.6 & 0 & -6.2 & 50.5 & -7.1 & -1.6 \\
\hline $3 \mathbf{h}$ & 23.1 & 0 & 0 & 35.4 & 0 & -5.4 & 37.0 & -2.8 & -1.6 \\
\hline $3 \mathbf{i}$ & 50.0 & 80.6 & 87.8 & 84.1 & 59.1 & -10.9 & 66.6 & -20.2 & 4.7 \\
\hline $3 \mathbf{j}$ & 45.0 & 64.5 & 87.8 & 84.1 & 92.0 & -13.2 & 53.2 & -38.8 & 3.1 \\
\hline $5 a$ & 0 & 0 & 0 & 35.4 & 0 & -3.9 & 31.7 & -19.3 & 6.3 \\
\hline $5 b$ & 42.3 & 38.7 & 30.8 & 73.0 & 33.3 & -3.9 & 31.7 & -10.2 & 7.8 \\
\hline $5 c$ & 7.7 & 0 & 0 & 30.8 & 0 & -10.1 & 69.3 & -8.9 & 9.4 \\
\hline Ref. & 99.0 & 100 & 100 & 99.9 & 100 & -7.6 & 62.6 & -12.8 & 8.6 \\
\hline
\end{tabular}

Ref. $=$ difenconazole 


\section{Conclusions}

Thirteen novel triazole analogs of difenoconazole containing 1,3-dioxolane rings have been synthesized. Their structures have been verified by ${ }^{1} \mathrm{H}-\mathrm{NMR}, \mathrm{IR}, \mathrm{MS}$ and $\mathrm{x}$-ray diffraction data and elemental analysis. Some of them display levels of plant-growth regulatory activity similar to those of a difenoconazole standard, but compared to the commercial agent their antifungal activities were not encouraging. It is possible that the existence of the methylene on the phenyl group destroys the conjugated system of the molecule, thus causing the lower fungicidal activities observed.

\section{Acknowledgements}

This work was supported by the National Natural Science Foundation of China (No. 20275020), the Natural Science Foundation of Shandong Province (No. Z2002B02) and the Outstanding Young Adult Scientific Research Encouraging Foundation of Shandong Province (No. 03BS081).

\section{Experimental}

\section{General}

IR spectra (KBr disks) were recorded on a Shimadzu-IR-435 Spectrophotometer. ${ }^{1} \mathrm{H}-\mathrm{NMR}$ spectra were recorded with a JEOL-ECP600 NMR Spectrometer $\left(\mathrm{CDCl}_{3}\right.$ as solvent, TMS as internal standard). Mass spectra were taken on a HP-5988A Spectrometer. Elemental analyses were determined on a Yanaco-CHNCORDER MT-3 automatic elemental analyzer. All agents were analytical grade and were used without further purification.

\section{Preparation of intermediates $\mathbf{1 .}$}

The intermediates 1 were prepared according to a literature procedure [16]. The substituted acetophenones were reacted with bromine in anhydrous ether in the presence of 1,4-dioxane. Five intermediates 1 were prepared in this manner: $\mathrm{R}^{1}=\mathrm{C}_{6} \mathrm{H}_{5}, 83.1 \%, 88 \sim 90^{\circ} \mathrm{C} ; \mathrm{Me}_{3} \mathrm{CC}_{6} \mathrm{H}_{4}, 83.7 \%$, $74 \sim 76^{\circ} \mathrm{C} ; 4-\mathrm{ClC}_{6} \mathrm{H}_{4}, 81.5 \% ; 104 \sim 106^{\circ} \mathrm{C} ; 2,4-\mathrm{Cl}_{2} \mathrm{C}_{6} \mathrm{H}_{3}, 75.3 \%, 91 \sim 93^{\circ} \mathrm{C} ; 2-\mathrm{ClC}_{6} \mathrm{H}_{4}, 76.7 \%, 83 \sim 84^{\circ} \mathrm{C}$.

\section{Preparation of intermediates 2 and $\mathbf{4}$.}

The intermediates $\mathbf{2} \mathbf{a} \sim \mathbf{2} \mathbf{j}$ and $\mathbf{4 a \sim 4} \mathbf{c}$ are prepared according to the literature method [17]. Their melting point data is listed in Table 2. 
Table 2. The melting points and yields of intermediates $\mathbf{2}$ and $\mathbf{4}$

\begin{tabular}{cccc}
\hline $\begin{array}{c}\text { Intermediate } \\
\text { No. }\end{array}$ & $\mathbf{R}^{\mathbf{1}}$ & $\mathbf{R}^{\mathbf{2}}$ & $\mathbf{m p} /{ }^{\circ} \mathbf{C}$ \\
\hline $\mathbf{2 a}$ & $\mathrm{Ph}$ & $\mathrm{H}$ & $110 \sim 112$ \\
$\mathbf{2 b}$ & $\mathrm{Ph}$ & $\mathrm{CH}_{3}$ & $76 \sim 78$ \\
$\mathbf{2 c}$ & $t-\mathrm{Bu}-\mathrm{Ph}$ & $\mathrm{H}$ & $79 \sim 81$ \\
$\mathbf{2 d}$ & $t-\mathrm{Bu}-\mathrm{Ph}$ & $\mathrm{CH}_{3}$ & $96 \sim 98$ \\
$\mathbf{2 e}$ & $p-\mathrm{Cl}-\mathrm{Ph}$ & $\mathrm{H}$ & $106 \sim 108$ \\
$\mathbf{2 f}$ & $p-\mathrm{Cl}-\mathrm{Ph}$ & $\mathrm{CH}_{3}$ & $88 \sim 90$ \\
$\mathbf{2 g}$ & $2,4-\mathrm{Cl} \mathrm{l}_{2} \mathrm{Ph}$ & $\mathrm{H}$ & $92 \sim 94$ \\
$\mathbf{2 h}$ & $2,4-\mathrm{Cl} \mathrm{P}_{2}-\mathrm{Ph}$ & $\mathrm{CH}_{3}$ & $83 \sim 85$ \\
$\mathbf{2 i}$ & $o-\mathrm{Cl}-\mathrm{Ph}$ & $\mathrm{H}$ & $141 \sim 143$ \\
$\mathbf{2 j}$ & $o-\mathrm{Cl}-\mathrm{Ph}$ & $\mathrm{CH}_{3}$ & $67 \sim 69$ \\
$\mathbf{4 a}$ & $p-\mathrm{Cl}-\mathrm{Ph}$ & - & $77 \sim 79$ \\
$\mathbf{4 b}$ & $2,4-\mathrm{Cl} l_{2}-\mathrm{Ph}$ & - & $89 \sim 91$ \\
$\mathbf{4 c}$ & $o-\mathrm{Cl}-\mathrm{Ph}$ & - & $97 \sim 99$ \\
\hline
\end{tabular}

General procedure for the synthesis of compounds $\mathbf{3 a \sim 3 \mathbf { j }}$ and $\mathbf{5 a} 5 \mathbf{5}$ (with the preparation of $\mathbf{3 a}$ given as an example).

DMF (25 g) and 1H-1,2,4-triazole (15 mmol) were placed in a $100 \mathrm{~mL}$ flask. After the latter was completely dissolved through stirring, sodium methoxide in methanol $(15 \mathrm{mmol}$, prepared with 15 mmol sodium and $10 \mathrm{~mL}$ anhydrous methanol) was added dropwise. The mixture was maintained at room temperature $\left(18^{\circ} \mathrm{C}\right)$ for $0.5 \mathrm{~h}$ and the methanol was then distilled off till the temperature of the mixture rose to $140^{\circ} \mathrm{C}$. After the mixture was cooled below $60^{\circ} \mathrm{C}$, intermediate $2 \mathbf{a}(15 \mathrm{mmol})$ and dry $\mathrm{KI}(0.1 \mathrm{~g})$ were added. The mixture was stirred under reflux for $3 \mathrm{~h}$, and then it was poured into water $(50 \mathrm{~mL})$ under vigorous stirring to give $4.3 \mathrm{~g}$ of crude precipitate that was collected by filtration and further purified by column chromatography (silica gel, using 3:1 v/v ethyl acetate-cyclohexane as the eluent) to afford $3.16 \mathrm{~g}(62.5 \%)$ of the target product 1-((2-(4-(benzyloxy)phenyl-1,3-dioxolan-2yl)methyl-1H-1,2,4-trizole (3a), as white crystals with mp 105 107 ${ }^{\circ} \mathrm{C}$; IR $\mathrm{cm}^{-1}: 1174,1136$ (C-O-C), $1249\left(\mathrm{CH}_{2}-\mathrm{O}-\mathrm{Ph}\right) ;{ }^{1} \mathrm{H}-\mathrm{NMR} \delta: 8.12$ (s, $\left.1 \mathrm{H}, \mathrm{Tr}-\mathrm{H}\right), 7.90$ (s, 1H, Tr-H), 6.89 7.43 (m, 9H, J=8.4Hz, Ar$\mathrm{H}), 5.05\left(\mathrm{~s}, 2 \mathrm{H}, \mathrm{PhCH}_{2}-\mathrm{O}\right), 4.47\left(\mathrm{~s}, 2 \mathrm{H}, \mathrm{CH}_{2} \mathrm{Tr}\right), 3.76\left(\mathrm{~m}, 4 \mathrm{H}, \mathrm{O}_{-} \mathrm{CH}_{2}-\mathrm{CH}_{2}-\mathrm{O}\right)$; Anal. Calc. for $\mathrm{C}_{19} \mathrm{H}_{19} \mathrm{~N}_{3} \mathrm{O}_{3}$ (337.38) C 67.64, H 5.68, N 12.45; Found: C 67.60, H 5.77, N 12.70. The thirteen title compounds, which include the ten compounds $\mathbf{3 a} \sim 3 \mathbf{j}$ and the three compounds $\mathbf{5 a} \sim 5 \mathbf{c}$ were synthesized in the same manner. 
1-((2-(4-(benzyloxy)phenyl)-4-methyl-1,3-dioxolan-2-yl) methyl)-1H-1,2,4-tiazole (3b): Yield 58.7\%; mp 111 113 ${ }^{\circ} \mathrm{C}$; IR cm ${ }^{-1}: 1171,1136$ (C-O-C), 1239 ( $\left.\mathrm{CH}_{2}-\mathrm{O}-\mathrm{Ph}\right) ;{ }^{1} \mathrm{H}-\mathrm{NMR} \delta: 9.16$ (s, $\left.1 \mathrm{H}, \mathrm{Tr}-\mathrm{H}\right), 8.90$ (s, 1H, Tr-H), 7.90 8.47 (m, 9H, J=8.7Hz, Ar-H), 6.06 (s, 2H, $\left.\mathrm{PhCH}_{2}\right), 5.43$ (d, 2H, $\left.\mathrm{CH}_{2} \mathrm{Tr}\right), 4.87 \sim 5.06$ (m, 1H, O-CH-C-O), 4.22 4.88 (m, 2H, O-CH2-C-O), 1.00 1.09 (s, 3H, $\left.\mathrm{CH}_{3}\right)$; Anal. Calc. for $\mathrm{C}_{20} \mathrm{H}_{21} \mathrm{~N}_{3} \mathrm{O}_{3}$ (351.41) C 68.36, H 6.02, N 11.96; Found: C 68.47, H 5.95, N 12.18.

1-((2-(4-(4-t-butylbenzyloxy) phenyl)-1,3-dioxolan-2-yl) methyl)-1H-1,2,4-trizole (3c): Yield 63.7\%; mp 112 114 ${ }^{\circ} \mathrm{C}$; IR cm ${ }^{-1}: 1171,1136$ (C-O-C), $1236\left(\mathrm{CH}_{2}-\mathrm{O}-\mathrm{Ph}\right) ;{ }^{1} \mathrm{H}-\mathrm{NMR} \delta: 8.11$ (s, 1H, Tr-H), 7.89 $(\mathrm{s}, 1 \mathrm{H}, \mathrm{Tr}-\mathrm{H}), 6.90 \sim 7.44(\mathrm{~m}, 8 \mathrm{H}, \mathrm{J}=8.5 \mathrm{~Hz}, \mathrm{Ar}-\mathrm{H}), 5.01\left(\mathrm{~s}, 2 \mathrm{H}, \mathrm{PhCH}_{2}-\mathrm{O}\right), 4.48\left(\mathrm{~s}, 2 \mathrm{H}, \mathrm{CH}_{2} \mathrm{Tr}\right)$, $3.76 \sim 3.78\left(\mathrm{~m}, 4 \mathrm{H}, \mathrm{O}-\mathrm{CH}_{2}-\mathrm{CH}_{2}-\mathrm{O}\right), 1.32\left(\mathrm{~s}, 9 \mathrm{H}, 3 \mathrm{CH}_{3}\right)$; $\mathrm{MS}(\mathrm{EI}): \mathrm{m} / \mathrm{z} 394\left[\mathrm{M}^{+}\right]$; Anal. Calc. for $\mathrm{C}_{23} \mathrm{H}_{27} \mathrm{~N}_{3} \mathrm{O}_{3}$ (393.49) C 70.21, H 7.18, N 10.31; Found: C 70.53, H 6.85, N 10.82.

1-((2-(4-(4-t-butylbenzyloxy)phenyl)-4-methyl-1,3-dioxolan-2-yl)methyl)-1H-1,2,4-trizole (3d): Yield 62.6\%; mp 83 85 ${ }^{\circ} \mathrm{C}$; IR cm ${ }^{-1}: 1174,1173$ (C-O-C), $1245\left(\mathrm{CH}_{2}-\mathrm{O}-\mathrm{Ph}\right) ;{ }^{1} \mathrm{H}-\mathrm{NMR} \delta: 8.12 \sim 8.16$ (d, $1 \mathrm{H}$, Tr-H), 7.90 (s, 1H, Tr-H), 6.90 7.47 (m, 8H, J=8.6Hz, Ar-H), 5.02 (s, 2H, $\left.\mathrm{PhCH}_{2} \mathrm{O}\right), 4.43 \sim 4.45$ (d, 2H, $\left.\mathrm{TrCH}_{2}\right), 3.87 \sim 3.96(\mathrm{~m}, 1 \mathrm{H}, \mathrm{O}-\mathrm{CH}-\mathrm{C}-\mathrm{O}), 3.03 \sim 3.84\left(\mathrm{~m}, 2 \mathrm{H}, \mathrm{O}-\mathrm{CH}_{2}-\mathrm{C}-\mathrm{O}\right), 1.33\left(\mathrm{~s}, 9 \mathrm{H}, 3 \mathrm{CH}_{3}\right)$, 1.05 1.33 (d, 3H, J=5.6Hz, $\mathrm{CH}_{3}$ ); Anal. Calc. for $\mathrm{C}_{24} \mathrm{H}_{29} \mathrm{~N}_{3} \mathrm{O}_{3}(407.22) \mathrm{C} 70.72, \mathrm{H} \mathrm{7.18,} \mathrm{N} \mathrm{10.32;}$ Found: C 70.68, H 7.10, N 10.18.

1-((2-(4-(4-chlorobenzyloxy)phenyl)-1,3-dioxolan-2-yl) methyl)-1H-1,2,4-trizole (3e): Yield 66.7\%; mp 137 139 ${ }^{\circ} \mathrm{C}$; IR cm ${ }^{-1}$ : 1170, 1133 (C-O-C), 1232 ( $\left.\mathrm{CH}_{2}-\mathrm{O}-\mathrm{Ph}\right) ;{ }^{1} \mathrm{H}-\mathrm{NMR} \delta: 8.12$ (s, 1H, Tr-H), 7.91 (s, 1H, Tr-H), 6.93 7.40 (m, 8H, J=8.8Hz,Ar-H), 5.03 (s, 2H, $\left.\mathrm{PhCH}_{2} \mathrm{O}\right), 4.48\left(\mathrm{~s}, 2 \mathrm{H}, \mathrm{TrCH}_{2}\right)$, 3.76 3.78 (m, 4H, J=7.0Hz, $\left.\mathrm{A}_{2} \mathrm{~B}_{2}, \mathrm{O}-\mathrm{CH}_{2}-\mathrm{CH}_{2}-\mathrm{O}\right)$; Anal. Calc. for $\mathrm{C}_{19} \mathrm{H}_{18} \mathrm{ClN}_{3} \mathrm{O}_{3}$ (371.1) C 61.44, $\mathrm{H}$ 4.89, N 11.32; Found C 61.50, H 4.85, N 11.28.

1-((2-(4-(4-chlorobenzyloxy)phenyl)-4-methyl-1,3-dioxolan-2-yl)methyl)-1H-1,2,4-trizole (3f): Yield 52.3\%; mp 115 117 ${ }^{\circ}$; IR cm ${ }^{-1}: 1172,1134$ (C-O-C); $1229\left(\mathrm{CH}_{2}-\mathrm{O}-\mathrm{Ph}\right)$; ${ }^{1} \mathrm{H}-\mathrm{NMR} \delta: 8.13 \sim 8.18(\mathrm{~d}, 1 \mathrm{H}$, Tr-H), $7.91 \sim 7.92$ (d, 1H, Tr-H), 6.93 7.42 (m, 8H, J=8.6Hz, Ar-H), 5.03 (s, 2H, PhCH $_{2}, 4.20 \sim 4.59$ (m, $\left.2 \mathrm{H}, \mathrm{CH}_{2} \mathrm{Tr}\right), 3.82 \sim 3.97$ (m, 1H, O-CH-C-O), 3.11 3.80 (m, 2H, O-CH2 $\left.-\mathrm{C}-\mathrm{O}\right), 1.09 \sim 1.42(\mathrm{~d}, 3 \mathrm{H}$, $\mathrm{J}=5.8 \mathrm{~Hz}, \mathrm{CH}_{3}$ ); Anal. Calc. for $\mathrm{C}_{20} \mathrm{H}_{20} \mathrm{ClN}_{3} \mathrm{O}_{3}$ (385.85) C 62.32, H 5.23, N 10.91; Found C 62.29, $\mathrm{H}$ 5.27, N 10.84 .

1-((2-(4-(2,4-dichlorobenzyloxy)phenyl)-1,3-dioxolan-2-yl)methyl)-1H-1,2,4-trizole (3g) : Yield 62.1\%; mp 136 138 ${ }^{\circ} \mathrm{C}$; IR cm ${ }^{-1}$; 1182, 1144 (C-O-C); 1235 ( $\left.\mathrm{CH}_{2}-\mathrm{O}-\mathrm{Ph}\right) ;{ }^{1} \mathrm{H}-\mathrm{NMR} \delta: 8.13$ (s, 1H, Tr-H), 7.91 $(\mathrm{s}, 1 \mathrm{H}, \mathrm{Tr}-\mathrm{H}), 6.95 \sim 7.43(\mathrm{~m}, 7 \mathrm{H}, \mathrm{J}=8.4 \mathrm{~Hz}, \mathrm{~J}=6.6 \mathrm{~Hz}, \mathrm{Ar}-\mathrm{H}), 5.12\left(\mathrm{~s}, 2 \mathrm{H}, \mathrm{PhCH}_{2} \mathrm{O}\right), 4.49(\mathrm{~s}, 2 \mathrm{H}$, $\mathrm{TrCH}_{2}$ ), 3.77 3.80 (m, 4H, J=7.0Hz, $\left.\mathrm{A}_{2} \mathrm{~B}_{2}, \mathrm{O}-\mathrm{CH}_{2}-\mathrm{CH}_{2}-\mathrm{O}\right)$; MS (EI): m/z 406 [M ${ }^{+}$; Anal. Calc. for $\mathrm{C}_{19} \mathrm{H}_{17} \mathrm{Cl}_{2} \mathrm{~N}_{3} \mathrm{O}_{3}(406.27) \mathrm{C} 56.17, \mathrm{H}$ 4.22, N 10.34; Found C 56.21, H 4.28, N 10.24. 
1-((2-(4-(2,4-dichlorobenzyloxy)phenyl)-4-methyl-1,3-dioxolan-2-yl) methyl)-1H-1,2,4-trizole (3h) : Yield 53.6\%; mp 110 112 ${ }^{\circ} \mathrm{C}$; IR cm ${ }^{-1}$ : 1176, 1141 (C-O-C), $1233\left(\mathrm{CH}_{2}-\mathrm{O}-\mathrm{Ph}\right) ;{ }^{1} \mathrm{H}-\mathrm{NMR} \delta: 8.13 \sim 8.18$ (d, 1H, Tr-H), 7.91 7.92 (d, 1H, Tr-H), 6.95 7.46 (m, 7H, J=8.4Hz, J=6.6Hz, Ar-H), 5.12 (s, 2H, $\mathrm{PhCH}_{2} \mathrm{O}$ ), 4.43 4.46 (m, 2H, CH $2 \mathrm{Tr}$ ), 3.82 3.98(m, 1H, O-CH-C-O), $3.11 \sim 3.98$ (m, 2H, O-CH $-\mathrm{C}-\mathrm{O}$ ), 1.09 1.14 (m, 3H, J=5.9 Hz, $\mathrm{CH}_{3}$ ); Anal. Calc. for $\mathrm{C}_{20} \mathrm{H}_{19} \mathrm{Cl}_{2} \mathrm{~N}_{3} \mathrm{O}_{3}(420.30)$ C 57.16, H 4.56, N 10.00; Found C 57.20, H 4.49, N 10.10.

1-((2-(4-(2-chlorobenzyloxy)phenyl)-1,3-dioxolan-2-yl) methyl)-1H-1,2,4-trizole (3i): Yield 52.4\%; mp 113 115 ${ }^{\circ}$; IR cm ${ }^{-1}: 1193,1159$ (C-O-C), $1236\left(\mathrm{CH}_{2}-\mathrm{O}-\mathrm{Ph}\right) ;{ }^{1} \mathrm{H}-\mathrm{NMR}$ 8: 8.14 (s, 1H, Tr-H), 7.92 (s, $1 \mathrm{H}, \mathrm{Tr}-\mathrm{H}), 6.97 \sim 7.42(\mathrm{~m}, 8 \mathrm{H}, \mathrm{J}=8.4 \mathrm{~Hz}, \mathrm{~J}=5.5 \mathrm{~Hz}, \mathrm{Ar}-\mathrm{H}), 5.17$ (s, 2H, $\left.\mathrm{PhCH}_{2} \mathrm{O}\right), 4.92\left(\mathrm{~s}, 2 \mathrm{H}, \operatorname{TrCH}_{2}\right)$, 3.75 3.80 (m, 4H, J=7.0Hz, $\mathrm{A}_{2} \mathrm{~B}_{2}, \mathrm{O}-\mathrm{CH}_{2}-\mathrm{CH}_{2}-\mathrm{O}$ ); Anal. Calc. for $\mathrm{C}_{19} \mathrm{H}_{18} \mathrm{ClN}_{3} \mathrm{O}_{3}$ (371.82) C 61.38, $\mathrm{H}$ 4.88, N 11.30; Found C 61.32, H 4.90, N 11.38.

1-((2-(4-(2-chlorobenzyloxy)phenyl)-4-methyl-1,3-dioxolan-2-yl) methyl)-1H-1,2,4-trizole (3j): Yield 35.8\%; mp 108 110 ${ }^{\circ}$; IR cm ${ }^{-1}: 1176,1138$ (C-O-C), $1235\left(\mathrm{CH}_{2}-\mathrm{O}-\mathrm{Ph}\right)$; ${ }^{1} \mathrm{H}-\mathrm{NMR} \delta: 8.14 \sim 8.19$ (s, $1 \mathrm{H}$, $\operatorname{Tr}-\mathrm{H}), 7.91$ (s, 1H, Tr-H), 6.98 7.44 (m, 8H, J=8.4Hz, J=6.2Hz, Ar-H), 5.17 (s, 2H, $\left.\mathrm{PhCH}_{2} \mathrm{O}\right)$, 4.43 4.47 (m, 2H, CH $2 \mathrm{Tr}$ ), 3.09 4.07 (m, 2H, O-CH $2-\mathrm{C}-\mathrm{O}), 3.98 \sim 4.07$ (m, 1H, O-CH-C-O), $1.08 \sim 1.14$ $\left(\mathrm{d}, 3 \mathrm{H}, \mathrm{J}=6.2 \mathrm{~Hz}, \mathrm{CH}_{3}\right)$; MS (EI) $386\left[\mathrm{M}^{+}\right], 388$ [M+2]; Anal Calc. for $\mathrm{C}_{20} \mathrm{H}_{20} \mathrm{ClN}_{3} \mathrm{O}_{3}$ (385.85) C 62.26, H 5.22, N 10.89; Found C 62.29, H 5.27, N 10.84 .

1-((2-(4-(4-chlorobenzyloxy)phenyl)-2-methyl-1,3-dioxolan-4-yl) methyl)-1H-1,2,4-triazole (5a): Yield 45.7\%; mp 132 134 ${ }^{\circ} \mathrm{C}$; IR cm ${ }^{-1}: 1176,1136$ (C-O-C), $1242\left(\mathrm{CH}_{2}-\mathrm{O}-\mathrm{Ph}\right)$;. ${ }^{1} \mathrm{H}-\mathrm{NMR} \delta: 8.24$ (s, $1 \mathrm{H}, \mathrm{Tr}-$ $\mathrm{H}), 7.96$ (s, 1H, Tr-H), 6.89 7.35 (m, 8H, J=8.7Hz, Ar-H), $5.02\left(\mathrm{~s}, 2 \mathrm{H}, \mathrm{PhCH}_{2} \mathrm{O}\right), 4.35 \sim 4.37$ (m, $2 \mathrm{H}$, $\mathrm{CH}_{2} \mathrm{Tr}$ ), 3.81 4.31 (m, 3H, J=8.8Hz, $\left.-\mathrm{CH}_{2} \mathrm{CH}-\right), 1.61$ (s, 3H, $\left.\mathrm{CH}_{3}\right) ; \mathrm{MS}(\mathrm{EI}) 386\left[\mathrm{M}^{+}\right], 388$ [M+2]; Anal Calc. for $\mathrm{C}_{20} \mathrm{H}_{20} \mathrm{ClN}_{3} \mathrm{O}_{3}$ (385.85) C 62.26, H 5.22, N 10.89; Found C 62.20, H 5.18, N 10.92.

1-((2-(4-(2,4-dichlorobenzyloxy)phenyl)-2-methyl-1,3-dioxolan-4-yl) methyl)-1H-1,2,4-triazole (5b) : Yield 53.8\%; mp 109 $111^{\circ} \mathrm{C}$; IR cm ${ }^{-1}: 1174,1140$ (C-O-C), $1230\left(\mathrm{CH}_{2}-\mathrm{O}-\mathrm{Ph}\right)$; ${ }^{1} \mathrm{H}-\mathrm{NMR} \delta: 8.24(\mathrm{~s}, 1 \mathrm{H}$, Tr-H), 7.91 (s, 1H, Tr-H), 6.90 7.42 (m, 7H, J=8.6Hz, Ar-H), 5.10 (s, 2H, $\left.\mathrm{PhCH}_{2} \mathrm{O}\right), 4.35 \sim 4.63$ (m, $\left.2 \mathrm{H}, \mathrm{CH}_{2} \mathrm{Tr}\right), 3.81 \sim 4.37$ (m, 3H, $-\mathrm{CH}_{2} \mathrm{CH}-$ ), 1.60 (s, 3H, $\mathrm{CH}_{3}$ ); Anal Calc. for $\mathrm{C}_{20} \mathrm{H}_{19} \mathrm{Cl}_{2} \mathrm{~N}_{3} \mathrm{O}_{3}(420.30)$ C 57.16, H 4.56, N 10.00; Found C 57.19, H 4.50, N 10.12.

1-((2-(4-(2-chlorobenzyloxy) phenyl)-2-methyl-1, 3-dioxolan-4-yl) methyl)-1H-1, 2,4-triazole (5c) : Yield 25.3\%; mp 130 132 ${ }^{\circ} \mathrm{C}$; IR cm ${ }^{-1}: 1176,1138$ (C-O-C), $1235\left(\mathrm{CH}_{2}-\mathrm{O}-\mathrm{Ph}\right) ;{ }^{1} \mathrm{H}$ NMR $\delta: 8.24(\mathrm{~s}, 1 \mathrm{H}$, Tr-H), 7.96 (s, 1H, Tr-H), 6.93 7.39 (m, 8H, J=8.8Hz, Ar-H), 5.15 (s, 2H, $\left.\mathrm{PhCH}_{2} \mathrm{O}\right), 4.35 \sim 4.38(\mathrm{~m}$, $2 \mathrm{H}, \mathrm{CH}_{2} \mathrm{Tr}$ ), 3.82 3.88 (m, 3H, $-\mathrm{CH}_{2} \mathrm{CH}-$ ), 1.60 (s, 3H, $\mathrm{CH}_{3}$ ); Anal. Calc. for $\mathrm{C}_{20} \mathrm{H}_{20} \mathrm{ClN}_{3} \mathrm{O}_{3}(385.85)$ C 62.32, H 5.23, N 10.91; Found C 62.28, H 5.20, N 10.93 


\section{References and Notes}

1. Elbe, H. L.; Buechcl, K. H., Luerssen, K. Ger Pat. 3144670, 1983.

2. Anderson, N.H. Proceedings of 5th International Congress of Pesticide Chemistry (IUPAC), Kyoto, Japan 1982, 345.

3. Shi, Y. N.; Fang, J. X.; Xu, L. Z. Chem. J. Chin. Univ. 1992, 13, 1084.

4. Skoets, C.; Kraehmer, H.; Baumert, D. Ger Pat. 3340 989, 1986.

5. Xu, L. Z.; Jiao, K.; Zhang, S. S. and Kuang, S. P. Bull. Kor. Chem. Soc. 2002, 23, 1699.

6. Cai, Y. J. Pesticides 1985, 5, 38.

7. Zhao, G. F.; Yang, H. Z. Chin. Chem. Bull. 1995, 6, 34.

8. Richard, M. J. Recent Advances in the Chemistry of Insect Control II; Royal Society of Chemistry: Cambridge, UK, 1990; p. 206.

9. Duan, J. C; Tang, H. X. Henan Chem. Ind. 2002, 6, 8.

10. CCDC 250980 contains the supplementary crystallographic data for this compound. These data can be obtained free of charge via www.ccdc.cam.ac.uk/conts/retrieving.html (or from the CCDC, 12 Union Road, Cambridge CB2 1EZ, UK; fax: +44 1223 336033; e-mail: deposit@ccdc.cam.ac.uk).

11. Li, Y. C.; Yue, M. X.; Dong, J. H.; Yu, W. Q.; Han, Y. F.; Li, B. W.; Li, S, Z. Acta Agric. BoreallSin. 1999, 14, 79.

12. Zhao, Z. R. Acta Scient. Nat. Univ. Nank. 1990, 4, 75.

13. Zhao, Z. R.; Huang, G. Q. Acta Bot. Sin. 1990, 32, 815.

14. Zheng, Q. L.; Zhang, Z. X.; Tang, C.C.; Zhao, Z. R. Acta Scient. Nat. Univ. Nank. 1995, $28,46$.

15. Fang, J.X.; Zhong, J.; Li, Z. M. and Liu W. Appl. Organomet. Chem. 2003, 17, 1.

16. Licke, F. F.; Burckhalter, J. H. J. Am. Chem. Soc. 1942, 64, 453.

17. Chen, X. F.; Kong, H. Q. Pesticides 1981, 6, 16.

Sample Availability: Available from the authors.

C 2004 by MDPI (http://www.mdpi.org). Reproduction is permitted for noncommercial purposes. 